Careful inspection of the mean values published in the paper show that it is feasible to take a series of garments from within a range and find that they perform in a satisfactory manner-that is, they provide linear graduation and are effective in terms of the particular aspect of venous function which we studied. Our critics, however, have failed to grasp the importance of the work if they do not appreciate that it is important that these criteria should extend throughout the range of garments and be applicable to any of the size ranges supplied by the manufacturer. Further study of the materials they quote ${ }^{2}$ shows that it was necessary for a range of stockings to be manufactured specifically for this study so that graduated compression could be achieved across the patient group.

We agree that further work is required, and want to make clear that our study was carried out independently of any manufacturer support; it was undertaken in the interest of applied physiology and the desire to improve the quality of care available to this often neglected group of patients based on objective data that are applicable to the individual subject.

JULIA V CORNWALL CAROLINE J DORE JOHN D LEWIS

Northwick Park Hospital

Harrow, Middlesex HA1 3UJ

1 Reddy PJ, Lamont P, Downes S, et al. An objective assessment of compression hosiery in deep venous insufficiency. Archives of the 14th World Congress of the International Union of Angiolog 1986;12:98.

2 Horner J, Fernandes J, Fernandes E, et al. Value of graduated compression stocking in deep venous insufficiency. $\mathrm{Br}$ Med $f$ 1980;280:820-1

\section{Leg ulcers}

SIR,-Dr P K Buxton (11 December, p 1542) perpetuates a myth about venous ulcer which helps to deny curative treatment for up to a quarter of patients-namely, that isolated saphenofemoral incompetence (primary long saphenous in his terminology) with normal deep veins is a rare cause. This is not the case. We found 20 patients (with 23 ulcers) in a series of 80 patients who had "venous" ulcers in this class. ${ }^{1}$ Sethia and Dark identified 17 ulcerated legs with primary varicosities out of a total of 60 studied. ${ }^{2}$ Though they were able to show that such limbs often had deep to superficial incompetence at other sites, the results of treatment showed that it was the saphenofemoral incompetence that mattered.

Because of these two studies with their very similar results we believe that up to a quarter of patients attending for treatment of a leg ulcer have the possibility of permanent cure by saphenofemoral ligation and that all patients with ulcers should have a proper evaluation by Doppler studies and if possible photoplethysmography. Varicose ulcers are still far too much Cinderellas of the surgical world at considerable financial cost to the health service.

Hugh DudLEY

\begin{abstract}
Academic Surgical Unit,
St Mary's Hospital,

London W2 1NY

1 Hoare MC, Nicolaides AN, Miles CR, et al. The role of primary varicose veins in venous ulceration. Surgery 1983;82:450.

2 Sethia KK, Darke SG. Long saphenous incompetence as a caus of venous ulceration. Brf Surg 1984;71:754-5.
on
\end{abstract}

\section{Comparison of two measures of waiting times}

SIR,-In the third of their series of articles on waiting times Dr B Don and colleagues (14 November, $p$ 1247) point out that the proportion of patients on a waiting list who, at some census date, have waited for more than one year will be greater than the proportion who actually do wait for longer than one year. This is a well known statistical effect caused by so called "length biased sampling" in which the probability of a sample unit (individual) being included in the sample or study is proportional to the magnitude (duration) of its value (the waiting time).

This effect crops up repeatedly in health service studies, as, for instance, when lengths of stay are estimated from a ward census (an example which, ironically, appeared to be missed by the examiners for the membership of the Faculty of Community Medicine) or durations of illness are estimated from those ill on an interview day (as might be done using general household survey data). In fact, subject to some distributional assumptions, formulas are easily derived for correcting for this bias.

On the other hand, there is a potential bias that cannot be accounted for owing to the fact that some patients who may be in a census day study of waiting times can never be in an admissions study because they were admitted as emergency cases, or died, or "went private" before being admitted or were never admitted for other reasons. Thus, in this instance, the two types of study actually address different populations. The authors referred to this as causing an "inflation" of waiting list statistics (7 November, p 1197). It is, of course, nothing of the sort. The fact that waiting times can be so long that some patients, legitimately entered on to the lists, become emergency cases, die, or are forced by unendurable pain or distress to "go private" is actually a deflation of admission statistics. Implying that waiting list statistics are "inflated" by these people is Orwellian doublespeak.

Medical Care Research Unit,

JON NICHOLL

Medical School,

\section{Cox DR. Renewal theory. London: Methuen, 1972.}

AUTHORS' REPLY,-We are well aware that the principles illustrated in our third paper on waiting lists are familiar to epidemiologists and statisticians. For this reason we referred to a standard textbook of epidemiology. Both $\mathrm{Mr} \mathrm{Nicholl} \mathrm{and} \mathrm{we}$ are referring to characteristics of prevalence data with wide application in epidemiological and health service studies. It is clear, however, that these characteristics are not generally considered in the use of waiting list statistics (and perhaps in the use of other health service statistics too). We seem to be in agreement on the need to draw attention to these points.

In the discussion of our second paper we covered possible reasons why the two measures of waiting list length may be discrepant. We included all the categories discussed by Mr Nicholl. We accept that the term "inflation" has a connotation that "discrepancy" does not. Our point was that the two sets of figures show a consistent discrepancy, a term which we use in the text, for which a number of explanations are possible. We point out that certain categories of patients on the waiting list will never appear in admission based statistics. Some of the patients in these categories will not appear in admission statistics because of the difficulties in obtaining a timely admission. As we say, more detailed studies than our own would be required to determine the contribution of each of several possible factors.

B Don

Oxford Regional Health Authority,

M J GOLDACRE

Oxford OX3 7LF
SIR,-Dr B Don and colleagues rightly point out that a prevalence or census estimate of waiting time will overestimate the proportion of patients who have waited for a long time.

However, Hospital Activity Analysis fails to record the waiting experience of an appreciable number of patients placed on our waiting lists such as those who move outside the district, are admitted as emergencies, or die while on the list; those who are referred to adjacent districts; and those who no longer want treatment because their condition has improved or their attitude to it has altered. The authors' previous paper $(7$ November, p 1197) suggested that these patients constituted about $28 \%$ of the waiting lists they reviewed; they are likely to form a large proportion of the patients waiting a long time, since these outcomes are more probable with increasing time on the list. Thus Hospital Activity Analysis underestimates waiting times. We should be concerned about the waiting times of these patient groups.

A cohort method of estimating waiting time provides the most accurate description of patients' waiting experience and does not suffer from the inflationary or deflationary inaccuracies of prevalence and retrospective methods. This cohort technique follows a group of patients placed on the waiting list over a defined time period until all have left it and records the waiting experience of every cohort member (including those omitted by Hospital Activity Analysis). This technique is also the most appropriate incidence based method for measuring individual chances and risks of particular outcomes-for example, the chance of having to wait over one year or being admitted as an emergency. This is because it provides the appropriate denominator for such risk calculations, unlike the Hospital Activity Analysis technique suggested by Dr Don and colleagues.

Unfortunately no direct measurement of cohort waiting times is performed nationally, though a retrospective cohort method was described by Williams et al, ${ }^{1}$ which would enable an individual's chances of having to wait for any given time period to be measured. Furthermore, the recent introduction of the Körner data sets will remove one of the main inaccuracies of this retrospective cohort method, caused by a mismatch between the two information sources used.

Alan Mordue

South Tees Health Authority,

Poole Hospital,

Nunthorpe,

Cleveland TS7 0NJ

Williams DRR, West RJ, Hagard S, Dias AFS. Waiting list monitoring using information from hospital activity analysis and SBH 203 returns. Community Med 1983;5:311-6.

SIR,-The findings of $\mathrm{Dr} M \mathrm{~J}$ Goldacre and colleagues (31 October, p 1105; 7 November, p 1197) highlight the complexities of compiling and interpreting waiting list statistics. We think that several other points should have been mentioned, particularly in their first article.

In our experience both cancellations and patients who do not arrive play a separate and important part in the waiting list equation and can therefore indirectly affect overall workload. The number of patients called for admission should therefore play a part in the calculation of waiting list statistics and trends.

Although in the reported study day cases have subsequently been removed from waiting list statistics in accordance with Department of Health and Social Security instructions, they represent a large proportion of the workload in some units and should therefore be audited and presented in a similar manner. Accurate waiting list statistics 